\title{
Application of Vortex Methods to Coaxial Rotor Wake and Load Calculations
}

\author{
Puneet Singh* and Peretz P. Friedmann ${ }^{\dagger}$ \\ University of Michigan, Ann Arbor, Michigan, 48109-2140
}

\begin{abstract}
The coaxial rotor has distinct advantages in hover performance and forward speed compared to a conventional isolated rotor helicopter. However, it experiences higher vibrations due to the unsteady aerodynamic interaction between the two rotors. The viscous vortex particle approach based on the Lagrangian formulation of the incompressible Navier-Stokes equations is a grid free method suitable for capturing the vortex wake interaction over long distances. It is applied to evaluate performance, wake evolution and loads in hover for a coaxial rotor system. A two dimensional analysis is used to evaluate the effects of blade pitch angle and vertical separation distance. It is found that the interaction is significant only for distances of less than 4 to 6 chord lengths between the airfoils. In the coaxial rotor analysis, the performance is sensitive to the viscous drag coefficient of the airfoil. The rational function approximation of unsteady airfoil loads was used to reduce computational effort and improve accuracy of the calculations. The performance and thrust oscillations were predicted well with both the methods.
\end{abstract}

\section{Nomenclature}

$\begin{array}{ll}{[\boldsymbol{A}]} & \text { self-induced aerodynamic influence matrix for airfoil } \\ b & \text { semi-chord } \\ \{\boldsymbol{b}\} & \text { normal velocity induced at airfoil control points by wake } \\ {[\boldsymbol{B}]_{(i, j)}} & \text { interactional aerodynamic influence matrix of } j^{\text {th }} \text { airfoil on } i^{t h} \text { airfoil } \\ c & \text { chord } \\ {\left[\boldsymbol{C}_{n}\right]} & \text { rational function approximation coefficient matrices } \\ C_{d} & \text { drag coefficient } \\ C_{l} & \text { lift coefficient } \\ C_{p} & \text { pressure coefficient } \\ d l & \text { differential length of airfoil surface panel } \\ {[\boldsymbol{D}],[\boldsymbol{E}],[\boldsymbol{R}]} & \text { matrices used in rational function approximation } \\ \{\boldsymbol{f}\} & \text { vector of generalized forces in the time domain } \\ \boldsymbol{F} & \text { force acting on airfoil } \\ \{\boldsymbol{G}\} & \text { laplace transform of load vector } \\ \{\boldsymbol{h}\} & \text { generalized airfoil motion vector } \\ \{\boldsymbol{H}\} & \text { laplace transform of generalized motion vector } \\ {[\boldsymbol{I}]} & \text { identity vector } \\ \boldsymbol{K} & \text { regularized Biot-Savart kernel } \\ M & \text { mach number } \\ n_{L} & \text { number of rational approximant poles } \\ N & \text { number of vortex particles } \\ \hat{\boldsymbol{n}} & \text { unit normal vector at airfoil surface } \\ p & \text { pressure } \\ {[\boldsymbol{Q}]} & \text { matrix transfer function relating generalized motions to aerodynamic loads } \\ \bar{s} & \text { laplace variable } \\ t & \text { airfoil velocity vector } \\ \boldsymbol{U} & { }^{*} \text { Ph.D. Candidate, Aerospace Engineering, Student Member AIAA } \\ \boldsymbol{v} & \end{array}$




$\begin{array}{ll}\text { Greek Symbols } & \\ \alpha & \text { particle vorticity } \\ \beta & \text { angle between point and vortex line } \\ \gamma_{\text {shed }} & \text { vorticity shed in the wake } \\ \gamma_{n} & \text { rational approximant pole } \\ \Gamma & \text { vector circulation of blade vortex filament } \\ \{\Gamma\} & \text { matrix vector of airfoil nodal circulations } \\ \zeta_{\sigma} & \text { radially symmetric particle vorticity distribution function } \\ \theta & \text { blade collective pitch angle } \\ \mu & \text { advance ratio } \\ \nu & \text { kinematic viscosity } \\ \rho & \text { density of air } \\ \sigma & \text { particle smoothing radius } \\ \phi & \text { potential due to vortex particles } \\ \omega & \text { vorticity, curl of velocity field }\end{array}$

Subscripts

$u \quad$ upper airfoil/rotor

$l \quad$ lower airfoil/rotor

\section{Introduction, Background and Objectives}

Despite a long history, the aerodynamic interactions in coaxial rotors are not well understood and high vibration levels are a source of concern. Reference [1] describes the progress in aerodynamic analyses conducted by various research groups till 1997. It compared experimental observations with momentum theory, blade element methods, free wake, prescribed wake methods and their combinations. It concluded that the structure of the wake and inflow distribution plays a critical role in the performance and dynamics of a coaxial helicopter.

Numerous studies have focused on using modified free wake analyses for coaxial configurations. A framework for a coaxial free wake method with good convergence characteristics has been considered [2]. The CHARM comprehensive rotorcraft analysis predicted good correlation with performance and wake geometry [3]. A time marching scheme [4] captured several vortex phenomena observed in experimental results. CAMRAD II was used for the analysis of coaxial rotors [5], and it also compared well with performance results. The Vortex Transport Model (VTM) was used for coaxial rotor analysis in Ref. [6]. The study predicted wake trajectories for various flight conditions and rotor interaction results correlated well with experiments. A high resolution CFD study [7] provided a detailed description of the flow field of the coaxial rotors. The results illustrated the azimuthal and radial variation of loads in hover, and good correlation with experimental performance data was found. CFD is the most accurate method for reproducing experimental data. It also produces an understanding of the nature of the flow field. However its practical implementation is challenging due to the extensive computational resources are required. Despite its accuracy, CFD is impractical for use in a comprehensive analysis code used for design or active blade control studies.

The Viscous Vortex Particle Method (VVPM) is an alternative to free wake method, VTM and CFD. VVPM directly solves the vorticity-velocity form of the incompressible Navier Stokes equations in the Lagrangian formulation. It does not require grid generation and can be coupled with lifting line models to obtain blade loads. VVPM has been applied to coaxial helicopter simulations in Refs. [8, 9]. The use of a hybrid CFD method [9] allows accurate modeling of viscous flow near the blades, and a non-dissipating rotor wake over large distances. Results from this study were reasonably close to experimental flow field measurements.

The accurate calculation of unsteady airfoil loads is essential for vibration prediction, control and analysis. A twodimensional unsteady aerodynamics reduced order model (ROM) has been used in vibration reduction studies for helicopters [10]. This ROM is based on the rational function approximation (RFA) of the frequency domain loads obtained from oscillatory response data using a two dimensional doublet-lattice analysis. Transformation to the time domain yields a state space model of the unsteady aerodynamic loads suitable for incorporation into comprehensive 


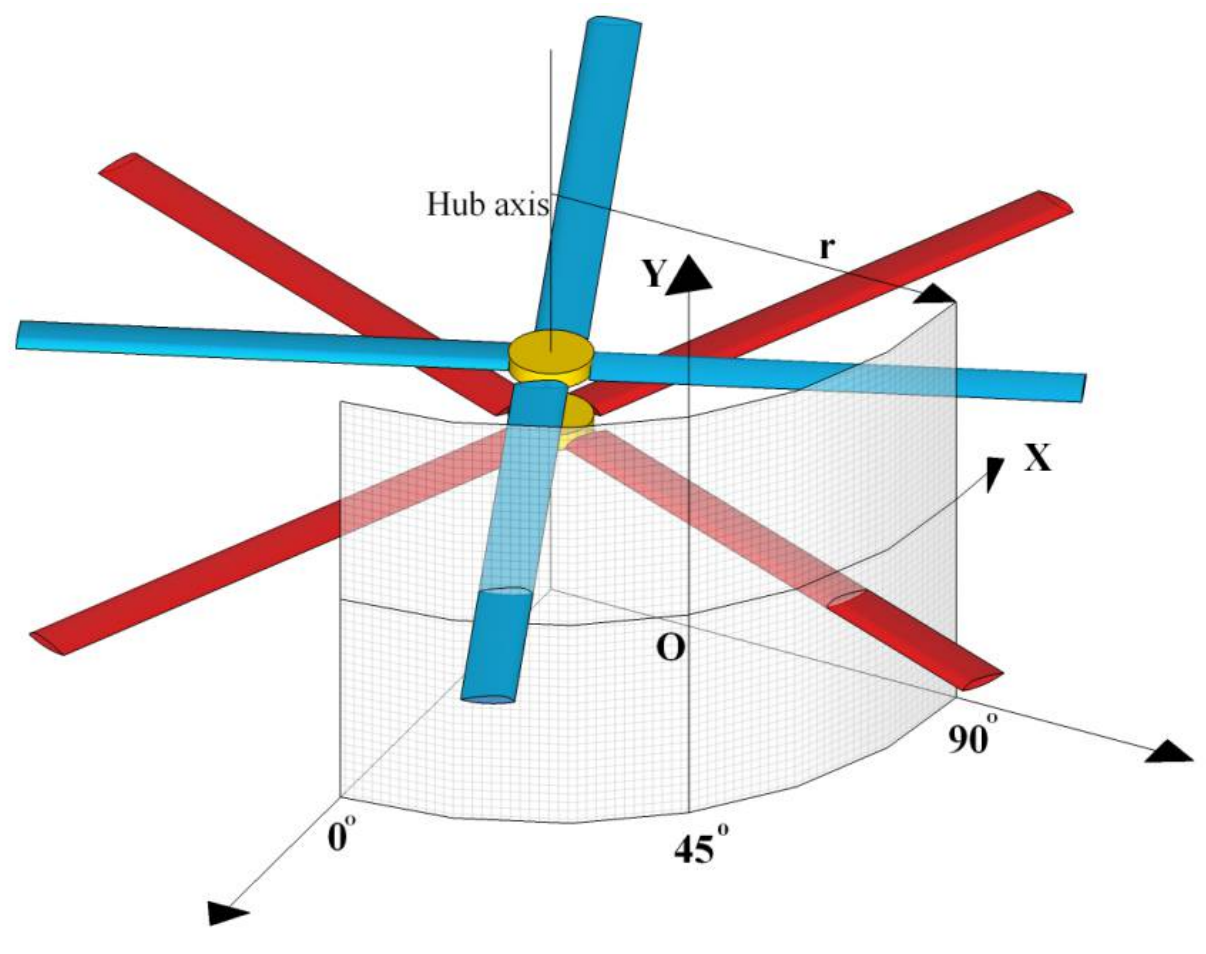

Figure 1. Extraction of 2D plane from coaxial rotor

rotorcraft simulation codes. This approach offers improved accuracy compared to the quasi steady lifting line method, and improved computational efficiency over CFD.

The overall objective of the paper is to apply the VVPM to the computation of wake and loads on coaxial rotors. The specific objectives of the paper are:

1. Describe a simplified two-dimensional vortex particle model for a coaxial rotor that illustrates interactional aspects.

2. Describe and validate the VVPM for a coaxial rotor simulation

3. Integrate RFA based unsteady aerodynamics with VVPM for rotor wake and load calculations.

\section{Problem Formulations}

\section{A. Two Dimensional Analysis}

Simplified coaxial rotor interaction can be illustrated by a two-dimensional (2D) problem consisting of two airfoils that pass over each other. This is described in Fig. 1 that illustrates a four-bladed coaxial rotor with NACA0012 airfoils. The upper rotor is shown in blue and rotates counterclockwise, while the lower rotor is shown in red and rotates clockwise. A 2D sectional plane is extracted along the arc shown in the figure. This arc is located at a radial location $r$ and extends from an azimuth of $0^{\circ}$ to $90^{\circ}$, such the blades pass each other at $45^{\circ}$ azimuth.

A vortex discretization of the airfoil is shown in Fig. 2. Each airfoil is modeled with $N+1$ nodal points distributed around the airfoil surface. Each nodal point is assigned a bound circulation. A total of $N$ control points are defined at the midpoints of every pair of consecutive nodes. The total normal velocity induced at each of the control points due to the bound circulation can be solved from a linear system of equations, using the Kutta condition.

$$
[\boldsymbol{A}]\{\boldsymbol{\Gamma}\}=\{b\}
$$




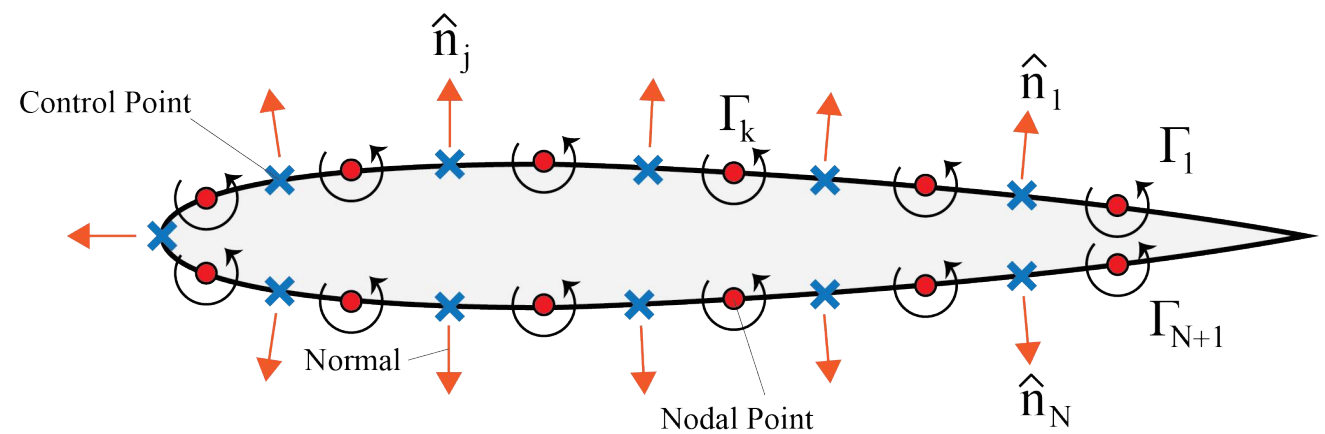

Figure 2. Vortex discretization of 2D NACA0012 airfoil

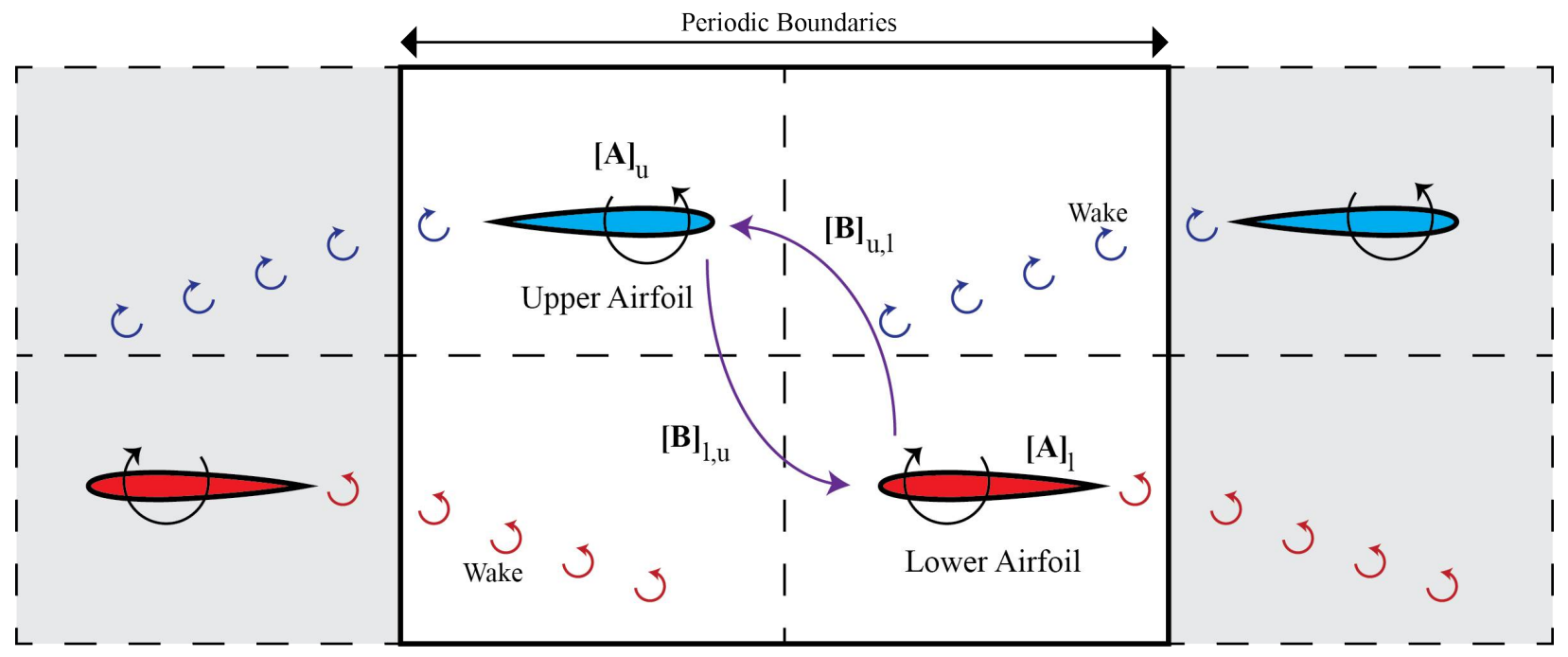

Figure 3. 2D cross-section of rotor blades indicating periodic boundaries and mutual interactional effects

The shed vorticity in the wake is obtained from the change in the circulation over the airfoil.

$$
\Gamma_{\text {shed }}\left(t_{i}\right)=\Gamma_{1}\left(t_{i}\right)+\Gamma_{N+1}\left(t_{i}\right)=\sum_{k=2}^{N} \Gamma_{k}\left(t_{i-1}\right)-\sum_{k=2}^{N} \Gamma_{k}\left(t_{i}\right)
$$

The net normal velocity due to the airfoil motion, bound vorticity and wake particles is set to zero at the control points, representing the non penetration boundary condition.

For identical blades in an axisymmetric hover situation, the 2D representation of an airfoil cross-section undergoes periodic motion and loading. To represent the periodicity, mirror images of the airfoil and its shed wake on both sides of the boundaries are used, as shown in Fig. 3. During the unsteady simulation, the airfoil self-aerodynamic influence matrices $[\boldsymbol{A}]$ remain invariant with time. The mutual aerodynamic influence matrices $[\boldsymbol{B}]$ are evaluated at each time step, because they depend on the relative displacement between the two airfoils. The influence of the wake system on the control points is captured by the $\{\boldsymbol{b}\}$ vectors. The following system of equations is solved:

$$
\left[\begin{array}{cc}
{[\boldsymbol{A}]_{u}} & {[\boldsymbol{B}(t)]_{u, l}} \\
{[\boldsymbol{B}(t)]_{l, u}} & {[\boldsymbol{A}]_{l}}
\end{array}\right]\left\{\begin{array}{l}
\left\{\boldsymbol{\Gamma}_{u}(t)\right\} \\
\left\{\boldsymbol{\Gamma}_{l}(t)\right\}
\end{array}\right\}=\left\{\begin{array}{l}
\left\{\boldsymbol{b}_{u}(t)\right\} \\
\left\{\boldsymbol{b}_{l}(t)\right\}
\end{array}\right\}
$$

Several passes of the airfoils are required to converge to a periodic solution. The $[\boldsymbol{B}]$ matrices need to be evaluated only during the first pass, and can be reused subsequently. 
The equation for the total force on a 2D airfoil is obtained by integrating the pressure field for potential flow over a solid surface in a multiply connected domain:

$$
\boldsymbol{F}=\underbrace{\left[\frac{\mathrm{d}}{\mathrm{d} t} \oint \rho \phi \hat{\boldsymbol{n}} \mathrm{d} l\right]}_{\text {Inertial }}+\underbrace{[\rho \boldsymbol{\Gamma} \times \boldsymbol{U}]}_{\text {Circulatory }}+\underbrace{\left[\rho \oint\left\{\frac{|\boldsymbol{v}|^{2}}{2}-(\boldsymbol{U} \cdot \hat{\boldsymbol{n}}) \hat{\boldsymbol{n}}\right\} \mathrm{d} l\right]}_{\text {Dynamic Pressure }}
$$

The first term represents the inertial effects on the thick airfoil, due to the change in potential. The second term is the circulatory lift, which depends on the total bound circulation on the airfoil. The third term depends on the dynamic pressure distribution. This term is zero for a domain with a single surface. Note that this is a potential flow solution therefore there is no viscous drag. However, components of the inertial and dynamic pressure terms contribute to the load in the direction of the drag.

\section{B. Vortex Particle Wake of Rotors}

The fundamental aspects of the VVPM are described in Refs.[11-13]. The vorticity-velocity form of the incompressible Navier-Stokes equations in the Lagrangian frame is:

$$
\frac{\mathrm{D} \boldsymbol{\omega}}{\mathrm{D} t}=\boldsymbol{\omega} \cdot \nabla \boldsymbol{u}+\nu \nabla^{2} \boldsymbol{\omega}
$$

The vorticity is discretized with particles of strength $\boldsymbol{\alpha}_{i}$ located at positions $\boldsymbol{x}_{i}$. The vorticity field can then be written as:

$$
\boldsymbol{\omega}(\boldsymbol{x}, t)=\sum_{i=1}^{N} \boldsymbol{\alpha}_{i}(t) \zeta_{\sigma}\left(\boldsymbol{x}-\boldsymbol{x}_{i}\right)
$$

The function $\zeta_{\sigma}$ is a radially symmetric regularization function. The subscript $\sigma$ is the smoothing radius of the vortex particle. For the current calculations, the gaussian kernel was selected:

$$
\zeta_{\sigma}(\rho)=\frac{1}{\sigma^{3}(2 \pi)^{3 / 2}} e^{-\frac{\rho^{2}}{2 \sigma^{2}}}
$$

Gaussian smoothing regularization functions and kernels were used in $[8,9,14,15]$. The flow field can be solved at any time $t$ by integrating the equations:

$$
\begin{gathered}
\frac{\mathrm{d} \boldsymbol{x}_{j}}{\mathrm{~d} t}=\sum_{\substack{i=1 \\
i \neq j}}^{N} \boldsymbol{K}_{\sigma}\left(\boldsymbol{x}_{j}-\boldsymbol{x}_{i}\right) \times \boldsymbol{\alpha}_{i}(t) \\
\frac{\mathrm{D} \boldsymbol{\alpha}_{j}}{\mathrm{D} t}=\boldsymbol{\alpha}_{j} \cdot \sum_{\substack{i=1 \\
i \neq j}}^{N} \nabla \boldsymbol{K}_{\sigma}\left(\boldsymbol{x}_{j}-\boldsymbol{x}_{i}\right) \times \boldsymbol{\alpha}_{i}(t)+\frac{2 \nu}{\sigma^{2}} \sum_{\substack{i=1 \\
i \neq j}}^{N}\left(V_{j} \boldsymbol{\alpha}_{i}-V_{i} \boldsymbol{\alpha}_{j}\right) \zeta_{\sigma}\left(\boldsymbol{x}_{j}-\boldsymbol{x}_{i}\right)
\end{gathered}
$$

Equations 8 - 9 convect the vortex particles and change their strength with time. The scheme can be shown to converge as the number of particles is increased. The error norms for the vorticity and velocity field decrease to zero, provided that the smoothing radius is larger than the distance between neighboring particles.

\section{Vortex Lines and Vortex Panels}

The bound vorticity on the lifting surface, such as a blade or a wing is represented with a vortex panel. A vortex panel is a collection of four connected vortex lines with identical circulation strength. The induced velocity and the associated vortex stretching is the summation of the contributions from each separate vortex filament. The velocity induced by a vortex line with a circulation strength $\Gamma$ at a point $P$ as shown in Fig. 4:

$$
\boldsymbol{u}_{\Gamma}(\boldsymbol{x}, t)=\sum_{i=1}^{N}\left(\boldsymbol{\Gamma}_{i} \times \boldsymbol{C P}\right)\left(\cos \beta_{1}+\cos \beta_{2}\right) \frac{1}{4 \pi|\boldsymbol{C P}|^{2}}
$$


The vortex stretching term due to a vortex line is:

$$
\begin{aligned}
& \boldsymbol{\alpha}_{k} \cdot\left(\nabla \boldsymbol{u}_{\Gamma}\right)=\quad \frac{\boldsymbol{\alpha}_{k} \times \boldsymbol{\Gamma}}{4 \pi|\boldsymbol{C P}|^{2}}\left(\cos \beta_{1}+\cos \beta_{2}\right) \\
& +\frac{\boldsymbol{\alpha}_{k} \cdot(\boldsymbol{\Gamma} \times \boldsymbol{C P})}{4 \pi|\boldsymbol{C P}|^{2}}\left[\hat{n}\left(\frac{1}{|\boldsymbol{A P}|}-\frac{1}{|\boldsymbol{B P}|}\right)-\frac{\boldsymbol{A P}}{|\boldsymbol{A P}|^{2}} \cos \beta_{1}-\frac{\boldsymbol{B P}}{|\boldsymbol{B P}|^{2}} \cos \beta_{2}\right] \\
& -2 \frac{\boldsymbol{\alpha}_{k} \cdot(\boldsymbol{\Gamma} \times \boldsymbol{C P})}{4 \pi|\boldsymbol{C P}|^{4}}\left(\cos \beta_{1}+\cos \beta_{2}\right) \boldsymbol{C P}
\end{aligned}
$$

\section{Panel Unsteady Load Calculation}

At each time step, the circulation over all the panels is calculated by solving the linear system of equations obtained by applying the no penetration condition at the panel collocation points. This is similar to the two dimensional approach described by Eqn. 3. However, the interaction terms are based on the 3D kernel. The $[\boldsymbol{A}]$ matrices remain invariant with time, while the $[\boldsymbol{B}]$ matrices are dependent on the relative orientation of the two rotors. Therefore, $[\boldsymbol{B}]$ matrices are periodic at the frequency of each revolution.

The pressure acting on a vortex panel can be calculated using the spatial gradient and time derivative of the circulation over the panel.

$$
\frac{\Delta p}{\rho}=\boldsymbol{v} \cdot\left(\frac{\mathrm{d} \Gamma}{\mathrm{d} x} \hat{\boldsymbol{n}}_{x}+\frac{\mathrm{d} \Gamma}{\mathrm{d} y} \hat{\boldsymbol{n}}_{y}\right)+\frac{\mathrm{d} \Gamma}{\mathrm{d} t}
$$

The Karman-Tsien mach correction factor is used to include compressibility effects at the local blade section. The Karman-Tsien compressibility correction is non-linear, and more accurate than the Prandtl-Glauert compressibility correction. The pressure coefficient at each panel is corrected based on the local Mach number:

$$
C_{p, \text { corrected }}=\frac{C_{p}}{\sqrt{1-M^{2}}+\frac{C_{p} M^{2}}{2 \sqrt{1-M^{2}}+2}}
$$

\section{Vorticity Generation}

The vorticity shed into the wake from a bound vortex line consists of two terms:

$$
\gamma_{\text {shed }}=-\frac{\mathrm{d} \boldsymbol{\Gamma}}{\mathrm{d} t}+\boldsymbol{u}_{b}(\nabla \cdot \boldsymbol{\Gamma})
$$

The first term represents the vorticity shed due to the time varying circulation on the lifting surface. The second term is the vorticity generated or released into the free stream due to the spanwise varying circulation.

Consider the blade panels in Fig. 5. The shed vorticity is represented by the particles aligned with the moving trailing edge. The trailing vorticity is represented by the particles emanating between two adjacent panels. They are the time varying and spanwise varying components of circulation over the panels respectively. Line vortices at the trailing edge ensure the net vorticity generated in the time step is zero.

\section{RFA unsteady aerodynamic loads}

In this approach, oscillatory response data of an airfoil is generated using two dimensional doublet-lattice analysis. The loads are approximated in the frequency domain as rational functions of the Laplace variable.

$$
\begin{gathered}
\{\boldsymbol{G}(\bar{s})\}=[\boldsymbol{Q}(\bar{s})]\{\boldsymbol{H}(\bar{s})\} \\
{[\boldsymbol{Q}(\bar{s})]=\left[\boldsymbol{C}_{0}\right]+\left[\boldsymbol{C}_{1}\right] \bar{s}+\sum_{n=1}^{n_{L}} \frac{\bar{s}}{\bar{s}+\gamma_{n}}\left[\boldsymbol{C}_{n+1}\right]}
\end{gathered}
$$

A state space model for the loads and aerodynamic states is generated by transforming the equations to time domain:

$$
\begin{array}{cc}
\{\dot{\boldsymbol{x}}\}= & \frac{U}{b}[\boldsymbol{R}]\{\boldsymbol{x}\}+[\boldsymbol{E}]\{\dot{\boldsymbol{h}}\} \\
\{\boldsymbol{f}\}=\frac{1}{U}\left(\left[\boldsymbol{C}_{0}\right]\{\boldsymbol{h}\}+\left[\boldsymbol{C}_{1}\right] \frac{b}{U}\{\dot{\boldsymbol{h}}\}+[\boldsymbol{D}]\{\boldsymbol{x}\}\right) \\
6 \text { of } 16
\end{array}
$$






Figure 4. Geometry of vortex line

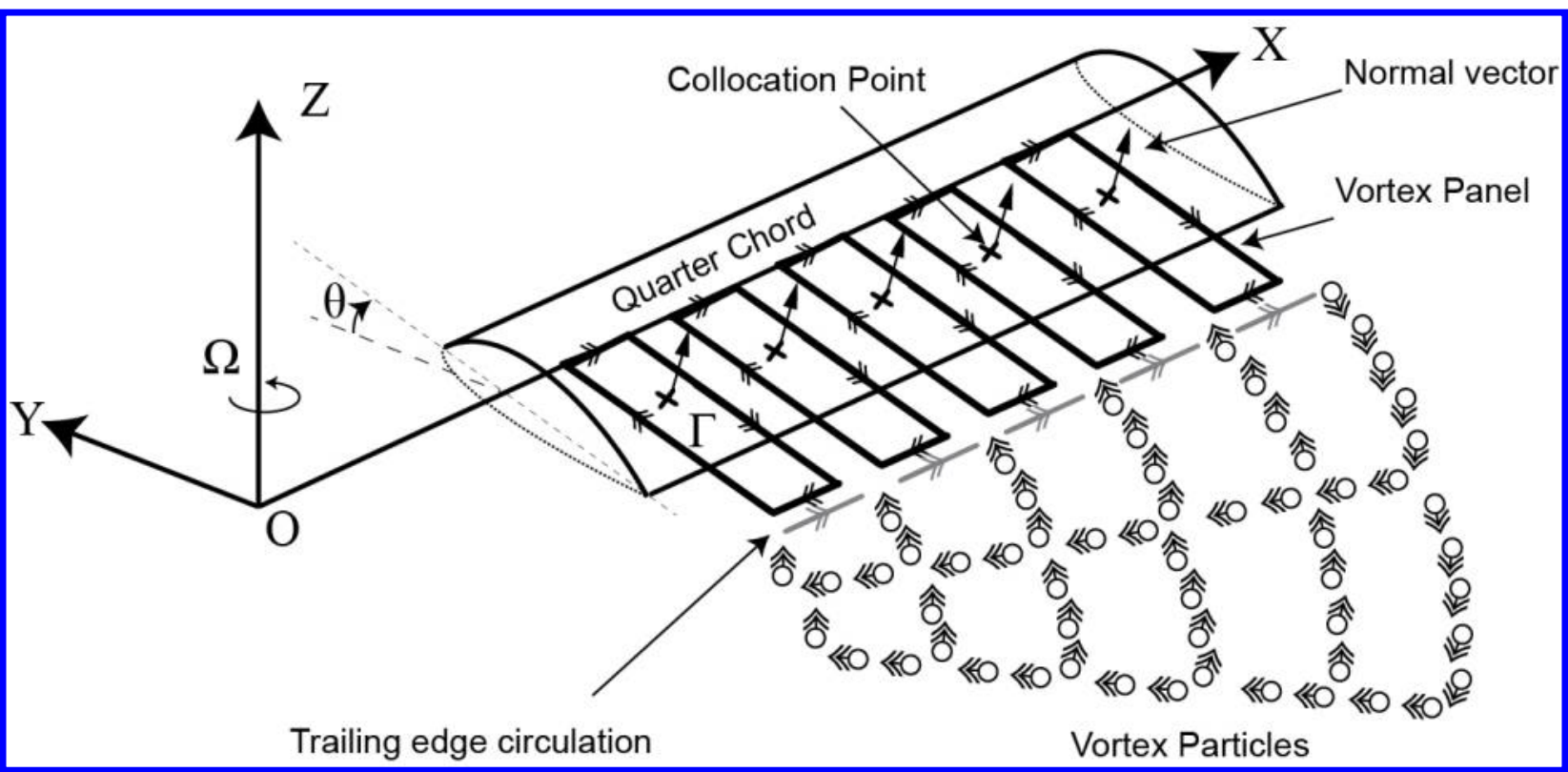

Figure 5. Schematic of vortex particle wake generation from a rotor blade

where:

$$
\begin{gathered}
{[\boldsymbol{D}]=[\boldsymbol{I} \cdots \boldsymbol{I}]} \\
{[\boldsymbol{E}]=\left[\begin{array}{c}
{\left[\boldsymbol{C}_{2}\right]} \\
\vdots \\
{\left[\boldsymbol{C}_{n_{L}+1}\right]}
\end{array}\right]} \\
{[\boldsymbol{R}]=-\left[\begin{array}{ccc}
\gamma_{1} \boldsymbol{I} & & \mathbf{0} \\
& \ddots & \\
\mathbf{0} & & \gamma_{n_{L}} \boldsymbol{I}
\end{array}\right]}
\end{gathered}
$$

The compressibility effects are included in the coefficient matrices for RFA approximation. The lift coefficient at each blade section can be transformed to the circulation of the panel using the transformation:

$$
\Gamma=\frac{c U C_{l}}{2}
$$




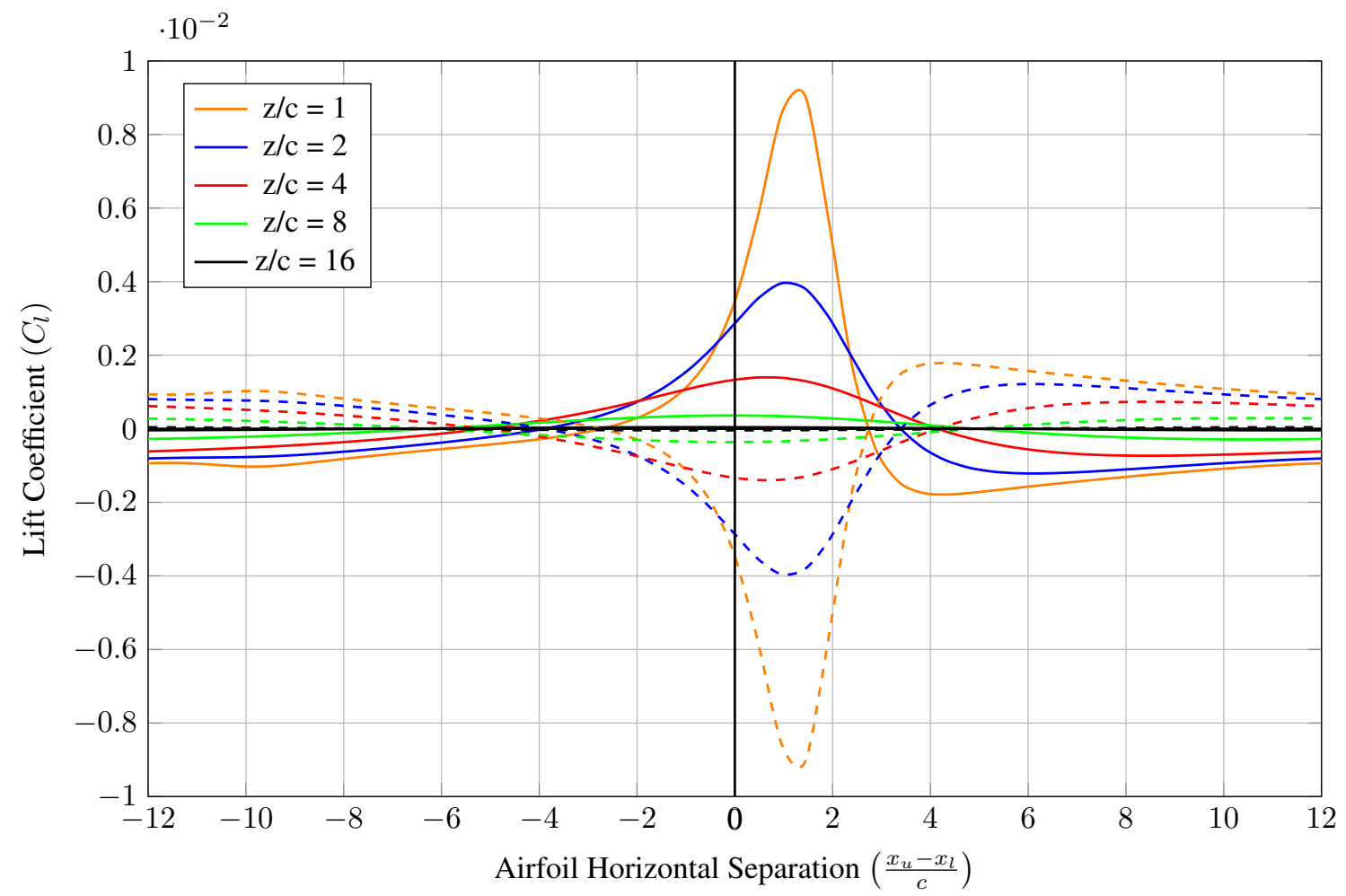

Figure 6. Change in Lift Coefficient for NACA0012 airfoils at $0^{\circ}$ blade pitch. Solid lines are for the upper airfoil, dashed lines are for the lower airfoil

An advantage of this method is the computational efficiency in the load calculations. In the vortex panel method, the interaction matrices $[\boldsymbol{A}]$ and $[\boldsymbol{B}]$ are evaluated. These matrices are fully populated and their size is determined by the total number of panels on all the rotor blades. At each time step, the linear system of equations (Eqn. 3) needs to be solved to obtain the circulation over the vortex panels. The computational complexity of the calculations is of $\mathcal{O}\left(n^{2}\right)$, where $n$ is the number of panels. Using the RFA methods for load calculations is computationally efficient because the generation of interaction matrices $[\boldsymbol{A}]$ or $[\boldsymbol{B}]$, and solving Eqn. 3 is not required. The computational complexity with the RFA model is $\mathcal{O}(n)$. Note that this complexity is separate from the VVPM calculations of the wake, which is $\mathcal{O}\left(N^{2}\right)$ where $N$ is the total number of vortex particles.

\section{Results}

\section{A. Two Dimensional Calculations}

The Sikorsky X2 rotor was used as a baseline case for simulating the coaxial rotor interaction, using the 2D plane approximately located at $0.75 R$. The maximum horizontal separation between the airfoils was 12 chord lengths. The NACA0012 airfoil was idealized with 101 nodal points and both airfoils were set to the same blade pitch angle. A range of vertical separations and blade pitch angles was tested. The vertical separation of two chord lengths represents the typical rotor spacing for the Sikorsky X2. Five mirror images of vortices on each side of the boundaries were used to simulate the periodicity. This corresponded to an effective maximum influence distance of 96 chord lengths for an image of a vortex, which was considered adequate. The results were found to reach a converged periodic state within 5-10 cycles.

Figure 6 illustrates the lift coefficient of each airfoil plotted as a function of their horizontal separation, based on Eqn. 4. Loads of the upper airfoil are shown in solid lines, while dashed lines indicate the lower rotor. The blade pitch angle was set to zero. While this implies a zero net thrust condition, it allows one to check for the symmetry of the aerodynamic environment and isolates airfoil thickness interaction effects. The symmetry of the interaction is evident in Fig.6. The change in lift is impulsive in nature, and occurs within a horizontal blade separation of 4 chord lengths. The lift on the upper airfoil increases, while it decreases for the lower airfoil. During the rest of the period the influence of the wake and blade produces a non-zero lift coefficient for the symmetric airfoil at zero pitch angle. 


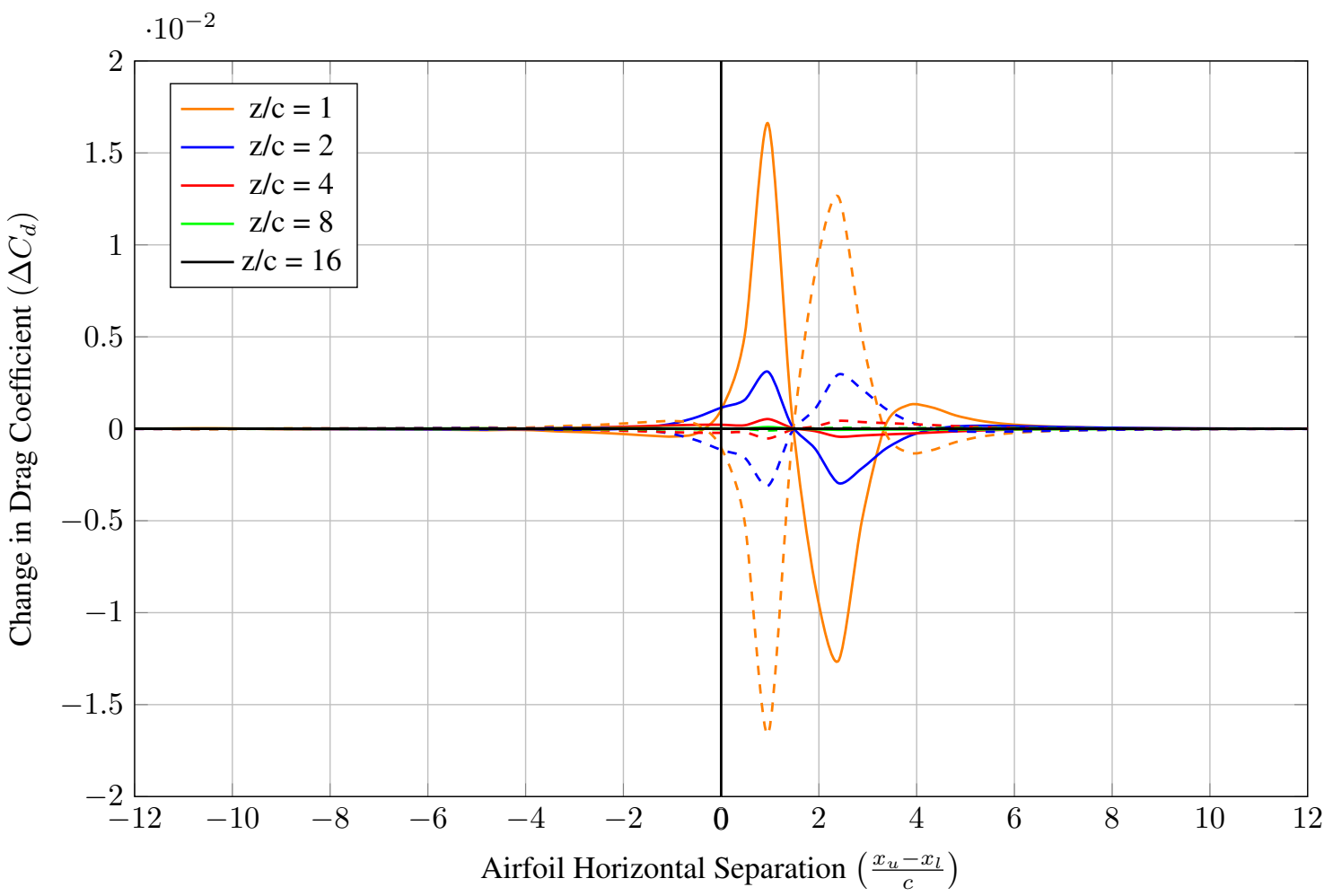

Figure 7. Change in Drag Coefficient for NACA0012 airfoils at $0^{\circ}$ blade pitch. Solid lines are for the upper airfoil, dashed lines are for the lower airfoil

However, due to the equal and opposite symmetric loads on each airfoil, the net thrust on the system is zero. The lift interaction is negligible beyond a vertical separation of 8 chord lengths.

The change in non-viscous drag is only significant after the airfoils have passed each other. The effect is limited to a short range of horizontal separation of approximately 4 to 6 chord lengths. The drag effect is negligible beyond a vertical separation of 8 chord lengths. For a vertical separation of 1 chord, the impulsive change in drag is of the same order of magnitude as the drag coefficient of the isolated NACA 0012 airfoil in steady ambient free stream conditions $\left(C_{d}=0.008\right)$.

The effect of blade pitch angle on the interaction was estimated by plotting the change in average circulatory lift coefficient during one period. The results for different blade pitch angles at a fixed vertical separation of 1 chord are shown in Fig.8. The magnitude of the oscillations, due to mutual interaction, increase with blade pitch angle. For blade pitch angles below $4^{\circ}$, the influence is strongest when the horizontal separation is less than 4 chord lengths. The upper airfoil also tends to have a larger lift coefficient compared to the lower airfoil in this region. However, at a pitch angle of $8^{\circ}$, difference in the loads is diminished. The upper airfoil has a higher lift coefficient as they approach each other. This effect is reversed as they move away. This indicates that the airfoil interactional loads are sensitive to the blade pitch angles, and not just the airfoil thickness and relative distance between them.

\section{B. VVPM Validation and Verification}

\section{Rotor Performance in Hover}

Rotor performance in hover is estimated using the thrust and torque coefficient. Rotor performance was verified with Ref. [16]. Two similar sized rotors were used in both the isolated and coaxial configuration each. The two rotors differed in the offset distance and the blade taper, as given in Tables 1 and 2. The thrust coefficient was plotted as a function of torque coefficient in Figs.9 and 10. The experimental data was compared with the blade element momentum theory results.

The rotor blades were modeled with two chordwise and eight spanwise panels, a configuration considered adequate 


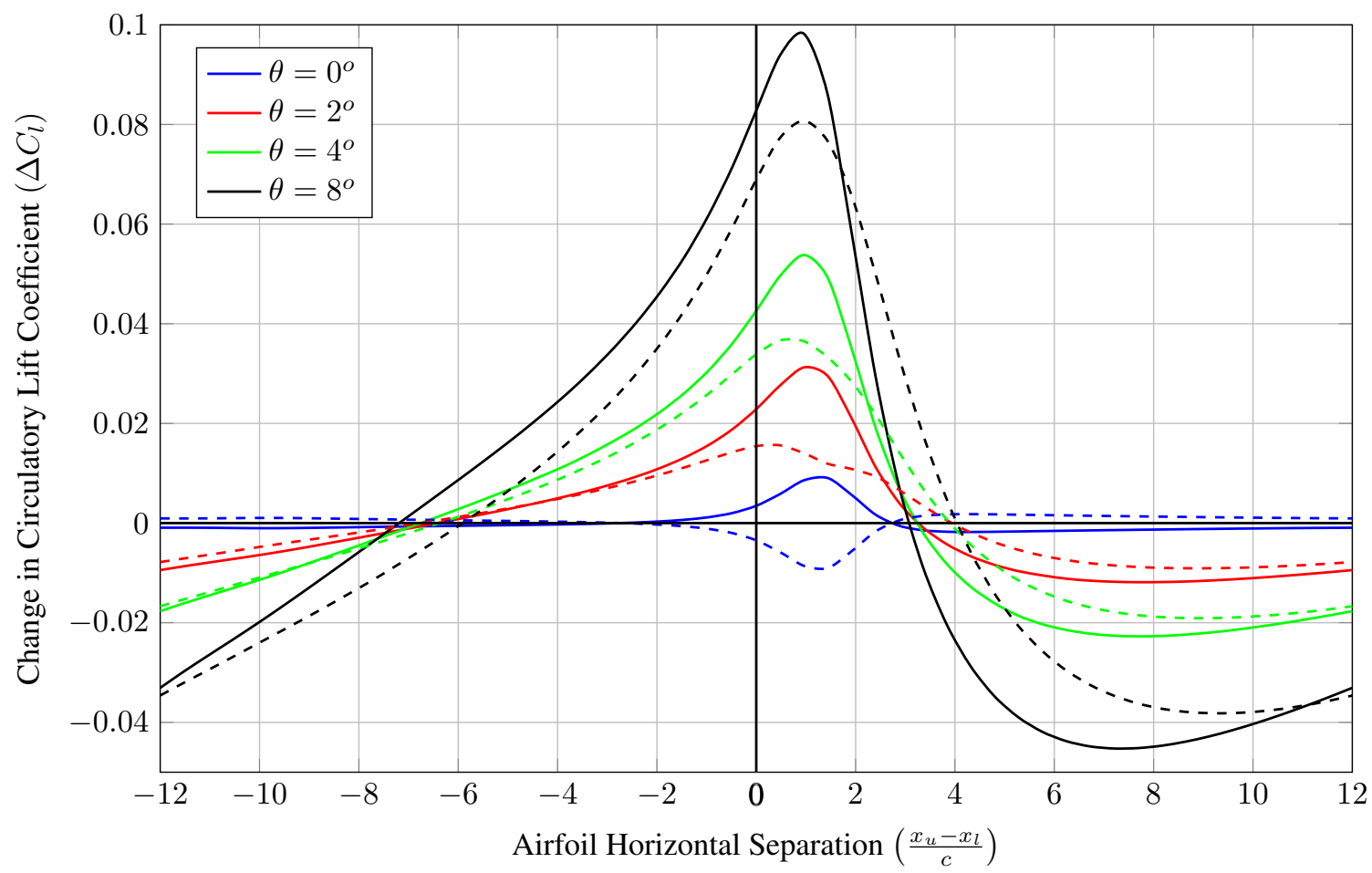

Figure 8. Change in Circulatory Lift Coefficient for NACA0012 airfoils at $z / c=1$ blade vertical separation. Solid lines are for the upper airfoil, dashed lines are for the lower airfoil

for this type of calculation. The periodicity in loads is achieved within 6 revolutions using an azimuthal step size of $12^{\circ}$. It was noted that the results do not change when using smaller step sizes. The total number of particles at the end of the simulations was approximately 8000, and the calculations were completed in less than 6 minutes on a desktop computer with 8 Intel Xeon(R) E5440 processors.

The two-dimensional drag coefficient at each blade section was assumed to have a quadratic dependence with the effective angle of attack:

$$
C_{d}=\delta_{0}+\delta_{1} \alpha+\delta_{2} \alpha^{2}
$$

The coefficients of the quadratic function were obtained by matching the torque coefficient at the zero thrust case for the rotor. A similar approach was used in several different studies, so as to resolve the drag discrepancy with experiments [16-18]. The modified drag coefficients are provided in Table 3.

In Figure 11, the rotor thrust and torque coefficient is calculated and compared with experimental data from Ref. [17]. For the isolated rotor, comparison is good. However, there is difference in the performance curve for the coaxial rotor. In the experiments performed in Ref. [17], the yaw trim was found to be sensitive to the collective settings. The yaw trim ensured that the net torque on the system was zero at a given total thrust by adjusting the collective pitch of the rotor. Reference [17] used a linear fit of imbalanced torque data and interpolation to obtain the zero torque condition. Therefore, the difference between the current calculations and available experimental data is attributed to the uncertainty of the yaw trim in the experiments.

\begin{tabular}{cc} 
Parameter & Value \\
\hline \hline Radius $(R)$ & $3.81 \mathrm{~m}$ \\
Chord $(c)$ & $0.4572 \mathrm{~m}$ \\
Offset $(e)$ & $0.508 \mathrm{~m}$ \\
Taper $\left(c_{\text {tip }} / c_{\text {root }}\right)$ & 0.3425 \\
Rotation Speed $(\Omega)$ & $31.36 \mathrm{rad} / \mathrm{s}$ \\
\hline \hline
\end{tabular}

Table 1. Rotor Parameters for Rotor 1 from Ref.[16] 


\begin{tabular}{cc} 
Parameter & Value \\
\hline \hline Radius $(R)$ & $3.81 \mathrm{~m}$ \\
Chord $(c)$ & $0.4572 \mathrm{~m}$ \\
Offset $(e)$ & $0.762 \mathrm{~m}$ \\
Rotation Speed $(\Omega)$ & $31.36 \mathrm{rad} / \mathrm{s}$ \\
\hline \hline
\end{tabular}

Table 2. Rotor Parameters for Rotor 2 from Ref.[16]

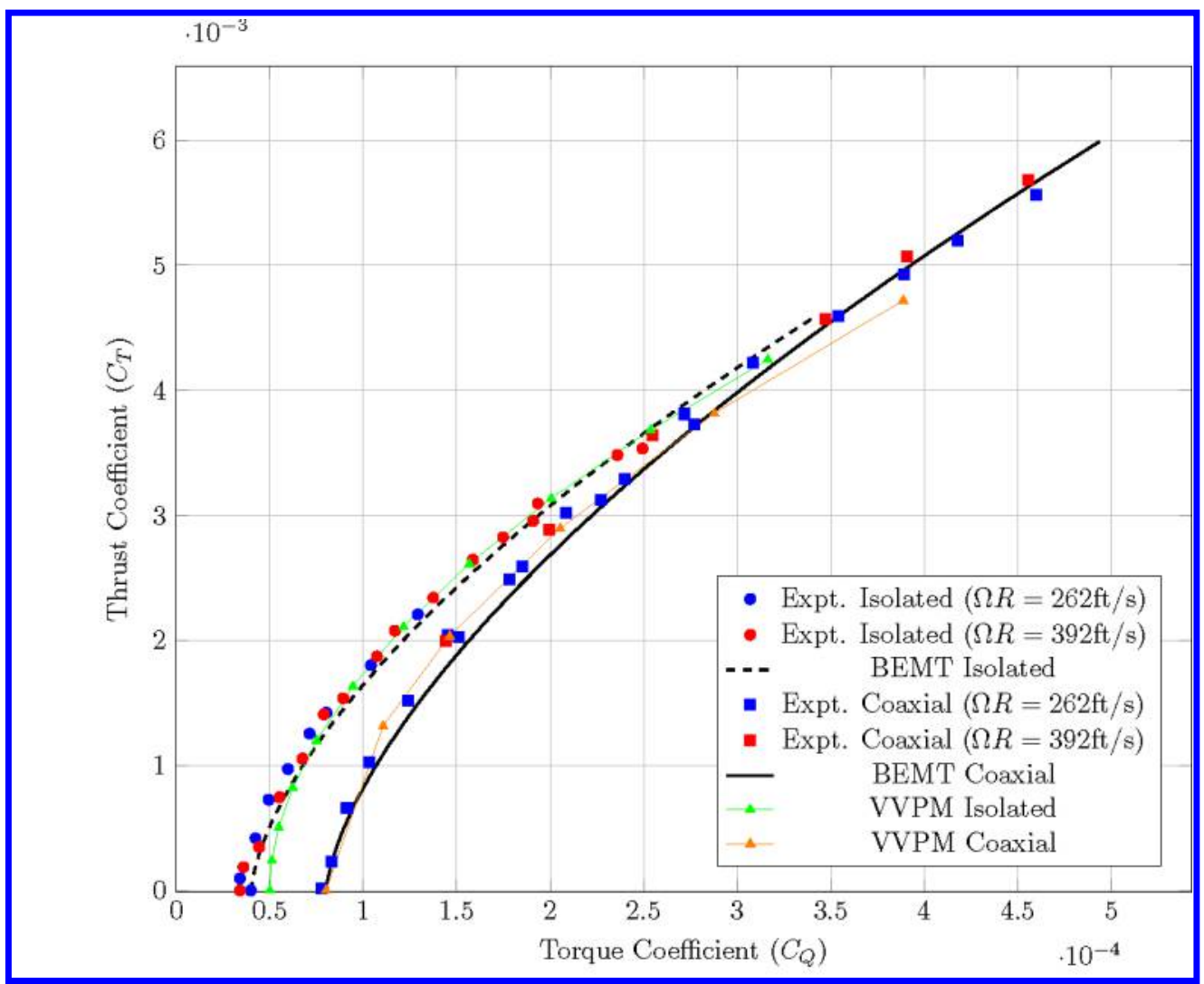

Figure 9. Comparison of experimental and computational thrust vs torque coefficient of Rotor 1 from Ref.[16]

\section{Calculation of Rotor Wake Boundary}

The visualization of the wake particles for highly twisted coaxial rotor blades from Ref. [19] is presented in Fig. 12. The blades were discretized into two chordwise and eight spanwise vortex panels. The helical descent of the shed vortices is evident in the figure. The vorticity is strongest at the tip, indicated by the orange particles. The inboard shed wake is blue because it has a vorticity strength that is about an order of magnitude smaller than the tip vortex particles. The tip vortex particles gradually lose strength due to vorticity stretching and viscous diffusion, which is evident from the yellow and green particles at lower levels of the wake.

In Fig. 13, the radial contraction of the tip vortex is plotted with respect to the vertical descent for isolated and coaxial rotors from Ref.[19]. The figure shows that the upper rotor wake contracts faster than the lower rotor. The lower rotor wake contraction is smaller when compared to the isolated case. The wake calculations indicate good qualitative agreement with wake visualizations provided in Ref. [19].

\section{Load calculation using RFA unsteady aerodynamics}

The rotor thrust and torque coefficient of the isolated and coaxial rotor from Ref. [17] was calculated using the RFA for unsteady aerodynamic loads in Fig. 14. A 6 pole approximation was used. The local mach number at the blade section was used to calculate the coefficient matrices used in the RFA equations. It is evident from Fig. 14 that the 


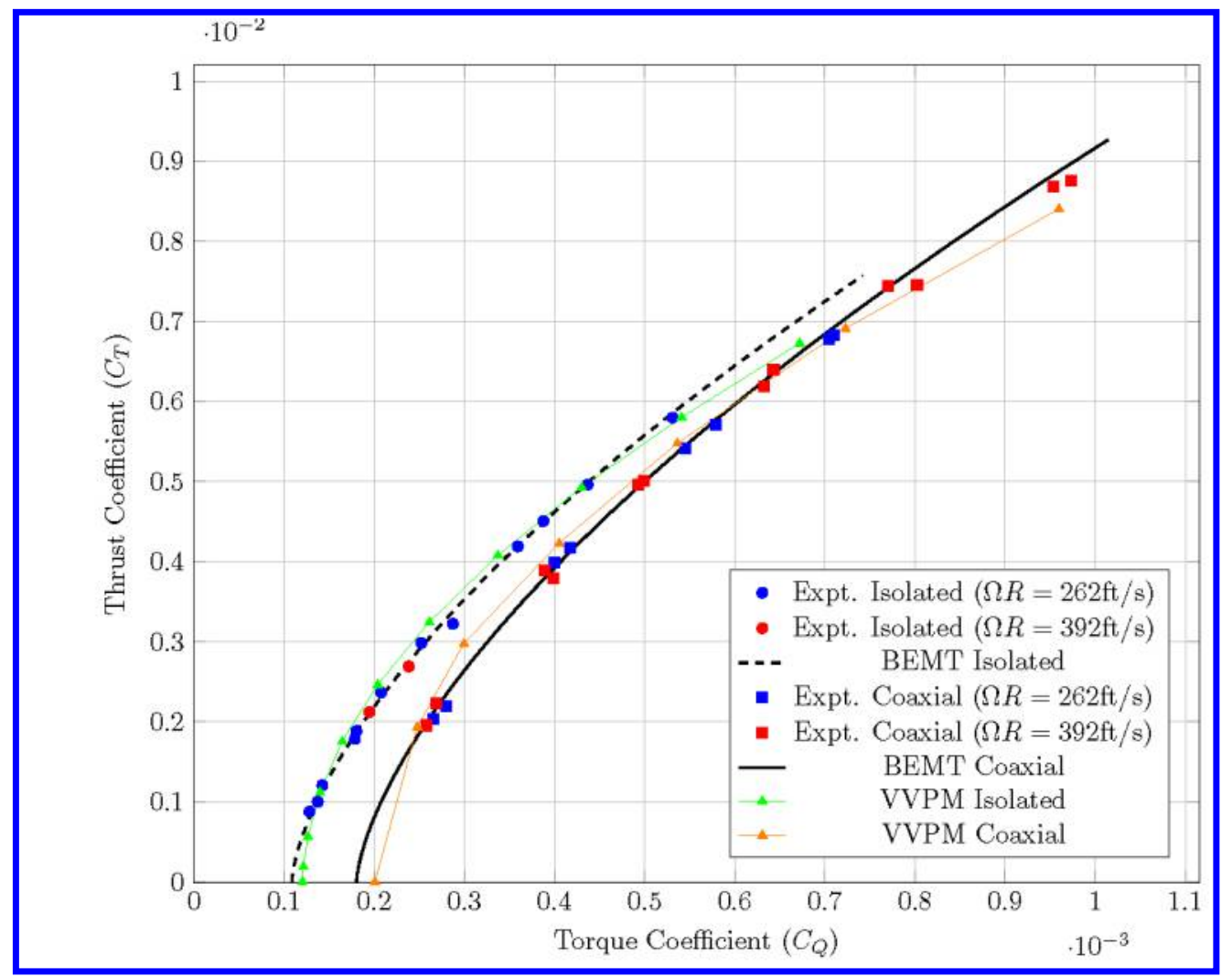

Figure 10. Comparison of experimental and computational thrust vs torque coeficient of Rotor 2 from Ref.[16]

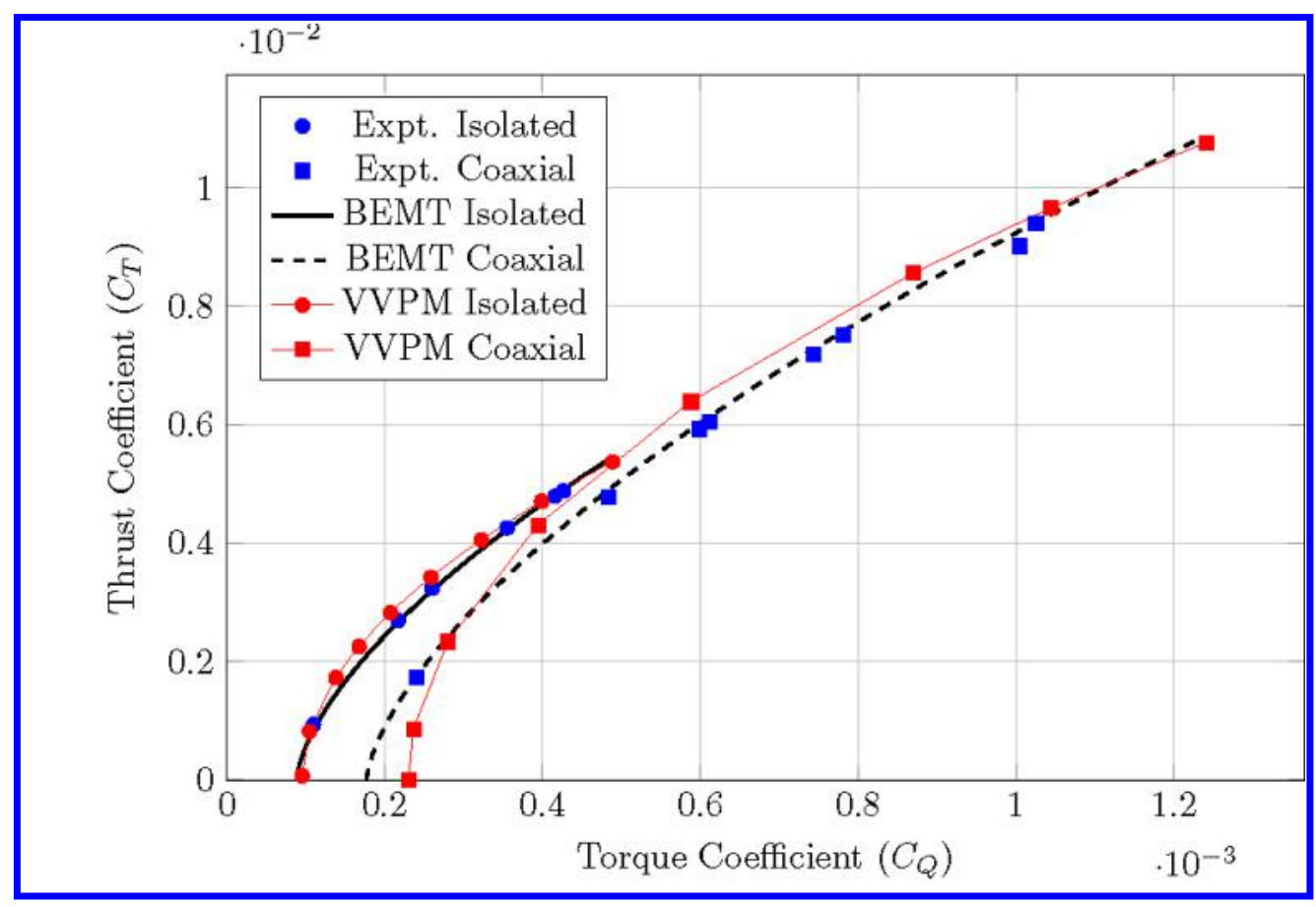

Figure 11. Comparison of experimental and computational thrust vs torque coefficient of Isolated and Coaxial Rotor for Twisted blades from Ref.[17] 


\begin{tabular}{cccccc} 
Rotor & Coefficient & BEMT Isolated & BEMT Coaxial & VVPM Isolated & VVPM Coaxial \\
\hline \hline \multirow{2}{*}{1} & $\delta_{0}$ & 0.009 & 0.009 & 0.005 & 0.004 \\
& $\delta_{1}$ & 0.0 & 0.0 & 0.0 & 0.0 \\
& $\delta_{2}$ & 0.8 & 0.8 & 0.3 & 0.5 \\
\hline \multirow{2}{*}{2} & $\delta_{0}$ & 0.0125 & 0.010 & 0.012 & 0.010 \\
& $\delta_{1}$ & 0.0 & 0.0 & 0.0 & 0.0 \\
& $\delta_{2}$ & 0.95 & 1.3 & 0.8 & 1.3 \\
\hline \hline
\end{tabular}

Table 3. Quadratic Drag Coefficients for Rotors

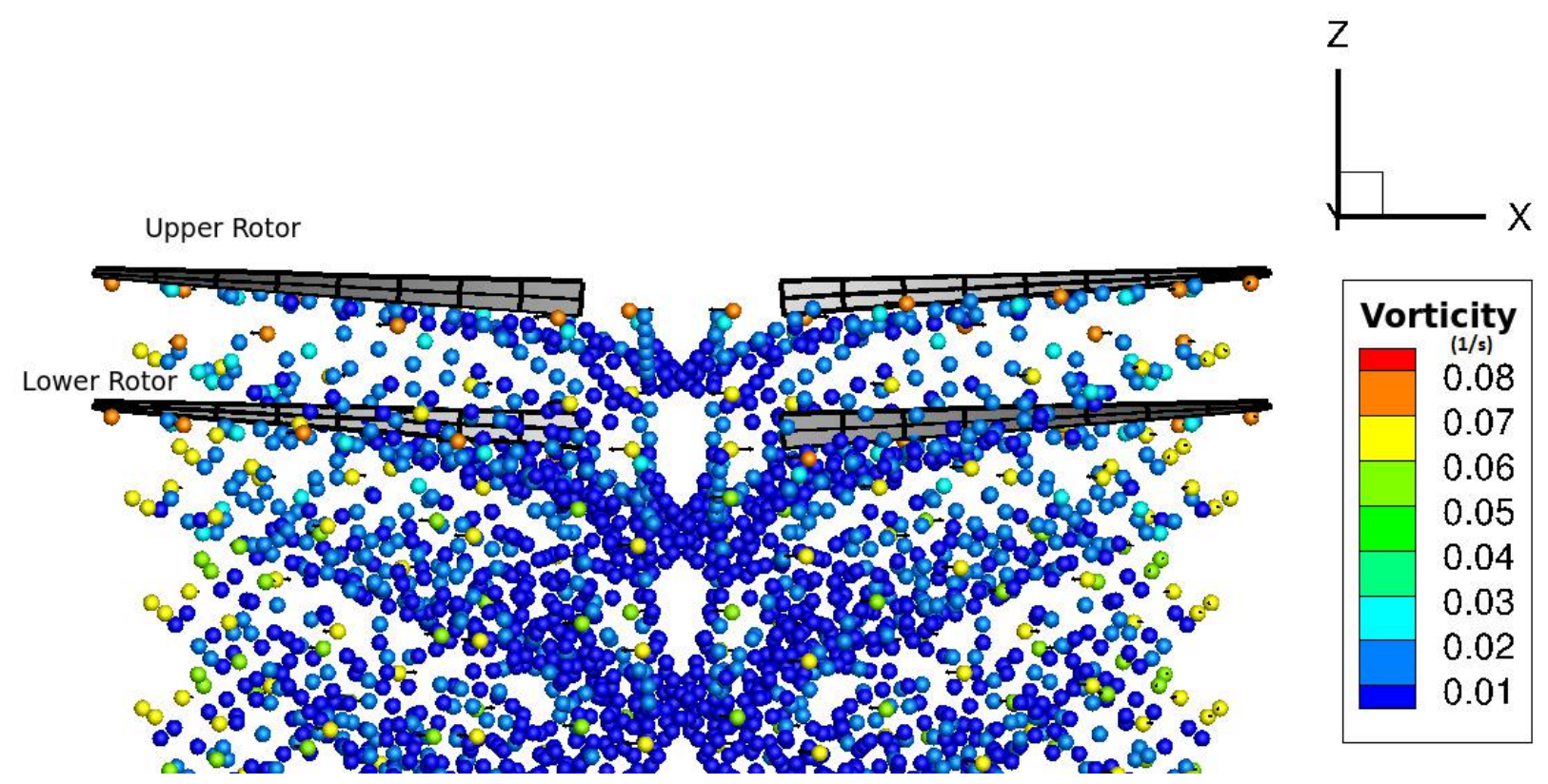

Figure 12. Particle wake visualization of coaxial rotors from Ref. [19] 


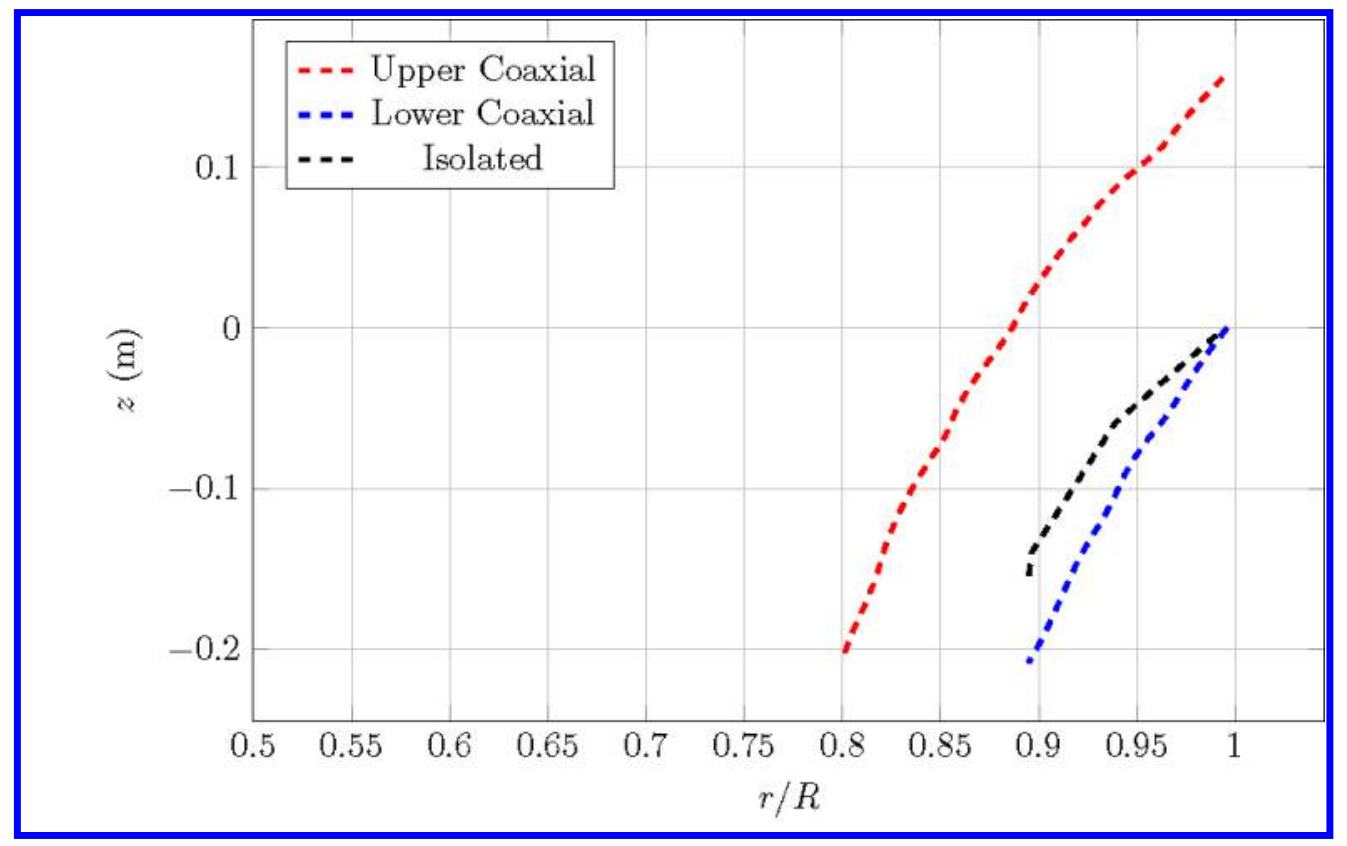

Figure 13. Tip vortex descent calculations of twisted rotor from Ref.[19]

RFA performance calculations are nearly identical to the vortex panel loads.

In Fig. 15, the unsteady vertical hub loads for the coaxial rotor of Ref. [17] are plotted. Both the load calculation methods indicate a dominant $4 / \mathrm{rev}$ component of vibratory loading. This is due to the blade overlap in the coaxial rotors that occurs at $0^{\circ}, 90^{\circ}, 180^{\circ}$ and $270^{\circ}$ azimuth. The vortex panel method calculations indicate a sharp increase in the loads at these azimuths. In comparison, the RFA method predicts a smaller magnitude of the load oscillations. This can be attributed to the damping due to inertial effects that is included in the RFA method. The vortex panel loads are higher because the circulation increases significantly when it is in close proximity to a passing tip vortex particle.

\section{Concluding Remarks}

The viscous vortex particle method was applied to isolated and coaxial rotors. A two dimensional analysis was conducted to understand the coaxial rotor interaction. In the complete rotor analysis, the blade lifting surfaces were represented by vortex panels. The rotor wake was simulated using VVPM. The RFA for unsteady aerodynamic loads was applied and compared with the vortex panel load results. The principal conclusions of this study are:

1. For the 2D analysis, the effect of interaction on the blade loads was confined to horizontal separation of 4 chords, and vertical separation of 8 chords. The impulsive change in drag occurred just after the airfoils had passed over each other. The upper airfoil had larger loads compared to the lower airfoil. This difference decreased at higher blade pitch angles.

2. In the vortex panel and VVPM analysis, the performance of isolated and coaxial rotors was validated by comparing the calculated results with experimental data. The ro tor performance was sensitive to the drag coefficient used for representation of airfoil section.

3. The rotor wake contraction calculations displayed good qualitative agreement with experimental flow visualization. The upper rotor wake contracted faster than the lower rotor wake. The lower rotor wake did not contract as fast as an isolated rotor wake.

4. The RFA unsteady aerodynamic loads model was combined with the VVPM for wake calculation. The rotor thrust and torque predictions were accurate. The vertical hub loads of the coaxial rotor indicated a dominant 4/rev vibration. The oscillations correspond to the azimuthal locations where the blades from the two rotors overlap each other. The magnitude of the oscillations is reduced with the RFA model, due to the inertial aerodynamic damping. 


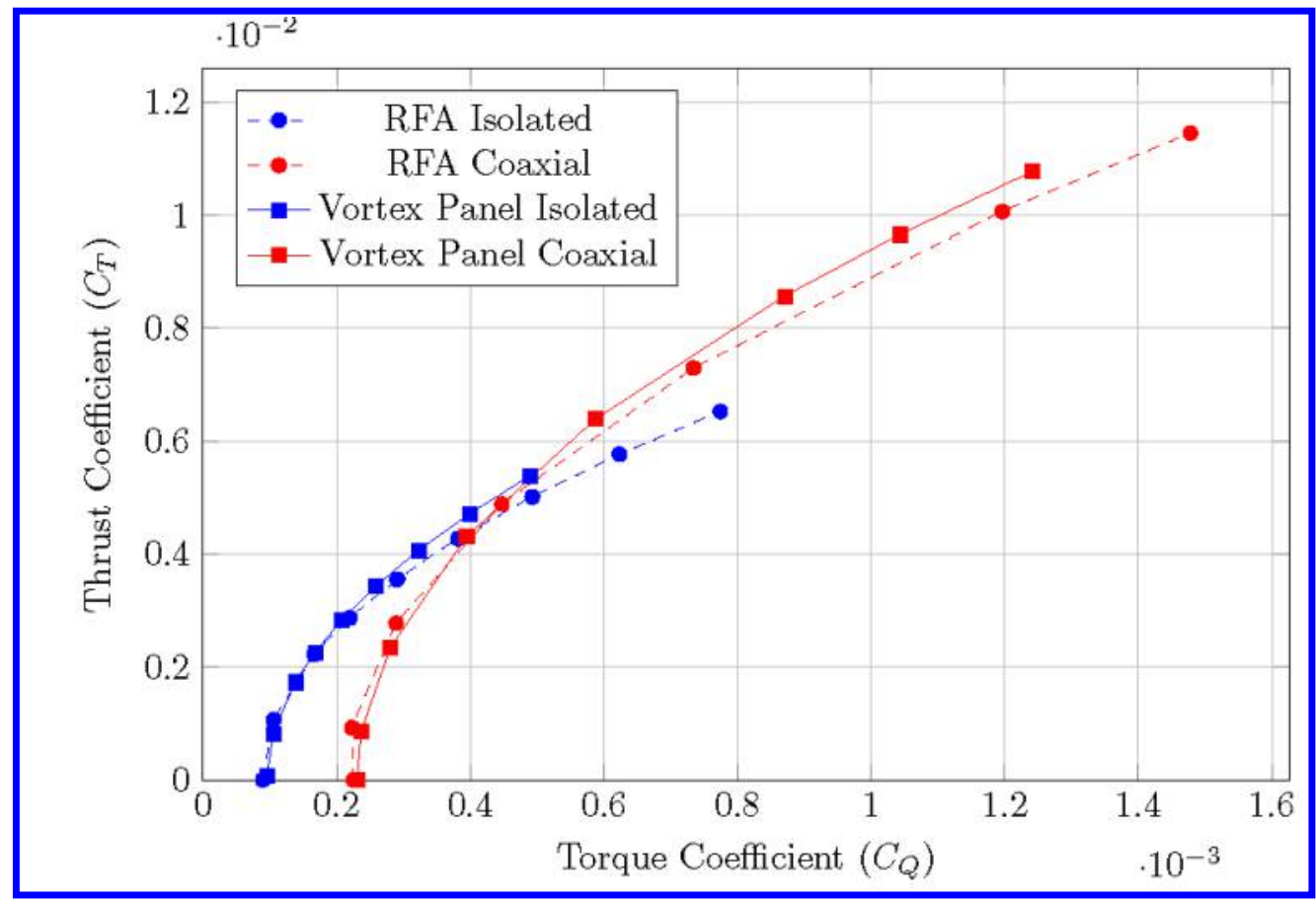

Figure 14. Thrust vs torque coefficient of rotor from Ref.[17] using Vortex Panel and RFA load calculations.

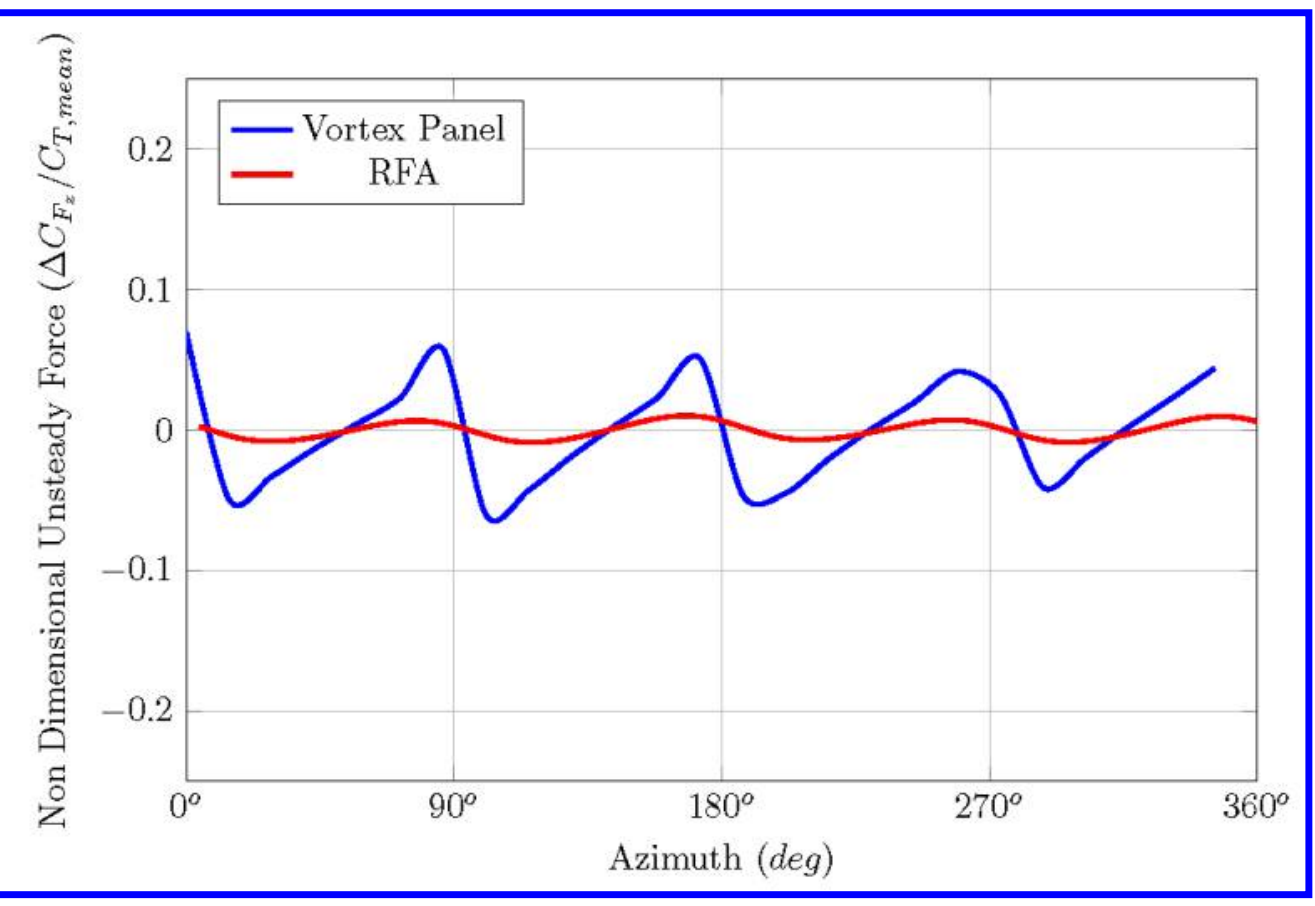

Figure 15. Comparison of unsteady vertical hub load vibrations for coaxial rotors using Vortex Panel and RFA load calculations 


\section{References}

[1] Coleman, C. P., “A survey of theoretical and experimental coaxial rotor aerodynamic research,” NASA Technical Paper 3675 , NASA Ames Research Center, Moffett Field, California, 1997.

-[2] Bagai, A. and Leishman, J. G., "Free-Wake Analysis of Tandem, Tilt-Rotor and Coaxial Rotor Configurations," Journal of the American Helicopter Society, Vol. 41, No. 3, 1996, pp. 196.

[3] Wachspress, D. A. and Quackenbush, T. R., "Impact of Rotor Design on Coaxial Rotor Performance, Wake Geometry and Noise," 62nd Annual Forum Proceedings-American Helicopter Society, Vol. 62, American Helicopter Society International, Inc, 2006, p. 41.

[4] Lee, J., Yee, K., and Oh, S., "Numerical Investigation of Dual Rotors Using a Time-Marching Free-Wake Method," 64th Annual Forum Proceedings-American Helicopter Society, Vol. 64, American Helicopter Society International, Inc., Montreal, Canada, 2008, p. 71.

- [5] Lim, J. W., McAlister, K. W., and Johnson, W., "Hover Performance Correlation for Full Scale and Model Scale Coaxial Rotors," Journal of the American Helicopter Society, Vol. 54, No. 3, 2009, pp. 32005.

[6] Kim, H. W. and Brown, R. E., "Coaxial Rotor Performance and Wake Dynamics in Steady and Manoeuvring Flight," 62nd Annual Forum Proceedings-American Helicopter Society, Phoenix, Arizona, 2006.

-[7] Lakshminarayan, V. K. and Baeder, J. D., "High-Resolution Computational Investigation of Trimmed Coaxial Rotor Aerodynamics in Hover," Journal of the American Helicopter Society, Vol. 54, No. 4, 2009, pp. 42008.

- [8] Zhao, J. and He, C., "Physics-Based Modeling of Viscous Ground Effect for Rotorcraft Applications," Journal of the American Helicopter Society, Vol. 60, No. 3, 2015, pp. 032006-1-13.

[9] Rajmohan, N., Zhao, J., and He, C., "A Coupled Vortex Particle / CFD Methodology for Studying Coaxial Rotor Configurations,” Fifth Decennial AHS Aeromechanics Specialists’ Conference, San Francisco, California, 2014.

-[10] Myrtle, T. F. and Friedmann, P. P., "Application of a new compressible time domain aerodynamic model to vibration reduction in helicopters using an actively controlled flap," Journal of the American Helicopter Society, Vol. 46, No. 1, 2001 , pp. 32-43.

-111] Cottet, G.-H. and Koumoutsakos, P. D., "Chapter 1 - Definitions and Governing Equations," Vortex Methods: Theory and Practice, Cambridge University Press, 2000, pp. 1-9.

[12] Saffman, P. G., Vortex dynamics, Cambridge University Press, 1992.

[13] Winckelmans, G. S. and Leonard, A., "Contributions to vortex particle methods for the computation of three-dimensional incompressible unsteady flows," Journal of Computational Physics, Vol. 109, No. 2, 1993, pp. 247-273.

-114] Zhao, J. and He, C., "A Viscous Vortex Particle model for Rotor Wake and Interference Analysis," Journal of the American Helicopter Society, Vol. 55, No. 1, 2010, pp. 12007-1-14.

[15] Zhao, J. and He, C., "Real-Time Simulation of Coaxial Rotor Configurations with Combined Finite State Dynamic Wake and VPM," American Helicopter Society 70th Annual Forum, Montreal, Canada, 2014.

[16] Harrington, R. D., "Full-scale-tunnel investigation of the static-thrust performance of a coaxial helicopter rotor," NACA Technical Note 2318, NACA Langley Aeronautical Laboratory, Langley Field, Virginia, 1951.

[17] Cameron, C. G., Karpatne, A., and Sirohi, J., "Performance and Vibratory Hub Loads of a Mach-Scale Coaxial Rotor in Hover," American Helicopter Society 70th Annual Forum, AHS International, Montreal, Canada, May 2014.

[18] Schmaus, J. and Chopra, I., "Performance and Loads Prediction for a High Advance Ratio Coaxial Rotor," AHS Forum 71, American Helicopter Society, 2015.

[19] McAlister, K. and Tung, C., "Experimental Study of a Hovering Coaxial Rotor with Highly Twisted Blades," 64th Annual Forum Proceedings-American Helicopter Society, Vol. 64, American Helicopter Society International, Inc., Montreal, Canada, 2008, p. 1765. 
This article has been cited by:

1. Natasha L. Schatzman, Narayanan Komerath, Ethan A. Romander. 2017. Time-Varying Loads of Co-Axial Rotor Blade Crossings. SAE International Journal of Aerospace 10:2. . [Crossref]

2. Han Han, Changle Xiang, Bin Xu, Yong Yu. Experimental and computational analysis of microscale shrouded coaxial rotor in hover 1092-1100. [Crossref] 